Doug Geisler, Eva K. Grebel, and Dante Minniti, eds.

\title{
HST Observations of 3 More Old LMC Clusters
}

\author{
Jennifer A. Johnson \\ OCIW, 813 Santa Barbara St., Pasadena, CA 91101, USA
}

Michael Bolte

UCO/Lick Observatory, UCSC, Santa Cruz, CA 95064, USA

Peter. B. Stetson \& James E. Hesser

DAO/HIA/NRC, 5071 West Saanich Rd., Victoria, BC, V8X 4M6, Canada

\begin{abstract}
We present color-magnitude diagrams (CMDs) based on HST F555W ("V") and F814W ("I") observations of three old LMC clusters: NGC 2210, NGC 1786, and Reticulum. The fiducial derived from the CMD of NGC 2257, another LMC cluster, provided a good fit to the data for the new clusters. Because NGC 2257 has a similar metallicity $([\mathrm{Fe} / \mathrm{H}] \sim-1.8)$ to NGC 2210 , NGC 1786, and Reticulum, the agreement between the CMDs of all four clusters indicates that they have the same age. This preliminary analysis suggests that any age differences are smaller than 2 Gyr. These new results mean that there are now 11 old LMC clusters with similar ages. An initial epoch of star cluster formation therefore happened in a short period over a large volume of space, a volume much larger than is now covered by the present-day optical LMC.
\end{abstract}

\section{Introduction}

The LMC provides a rare opportunity to study extragalactic clusters using the same methods as are used for the Milky Way clusters. Here we present colormagnitude diagram (CMD) analysis for three of the LMC clusters that are thought to be analogues of the old Galactic globular clusters. Previous work has established that the oldest LMC clusters are coeval with the old Galactic globular clusters such as M92. Olsen et al. (1998) used HST data to obtain the first deep CMDS for the inner $\left(<4^{\circ}\right.$ from center of LMC) metal-poor clusters and discovered that they were the same age as Milky Way globular clusters with similar metallicities, with little age spread among the clusters. Johnson et al. (1999) used HST data to argue that the clusters NGC 1466, NGC 2210 and Hodge 11 were coeval with each other and with the Milky Way clusters. Thus the metal-poor clusters studied so far in the LMC seemed to have been formed within a short period ( $<1.5 \mathrm{Gyr}$ ) period. Do Reticulum, NGC 1786, and NGC 2210 have similar ages? These three clusters, presented here, are metalpoor $([\mathrm{Fe} / \mathrm{H}] \sim-1.8 \mathrm{dex})$ in the outskirts of the LMC (Olszewski et al. 1991). 


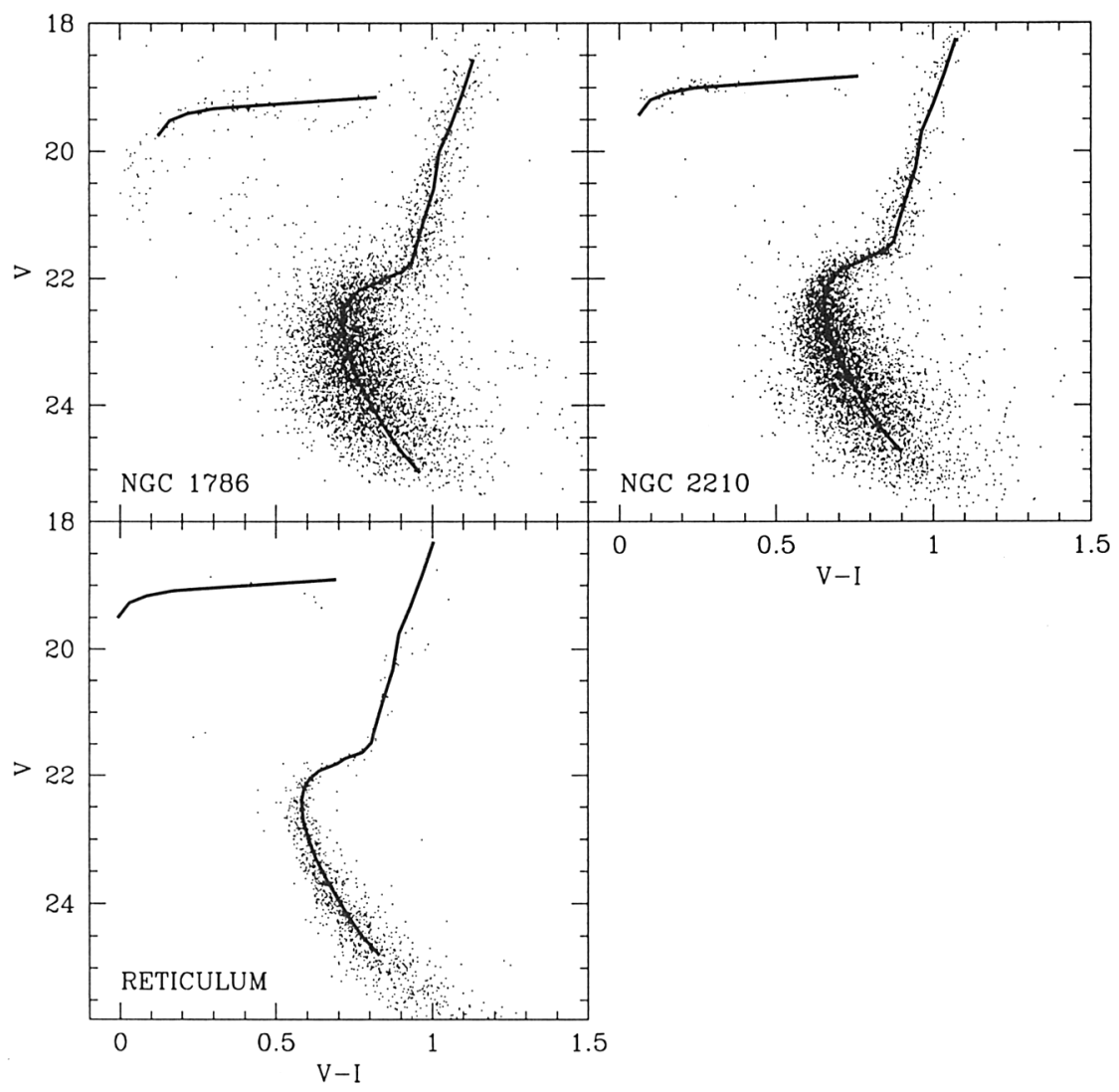

Figure 1. The $V, V-I$ color-magnitude diagrams for NGC 1786, NGC 2210, and Reticulum. Plotted on top of each cluster's data is the fiducial for NGC 2257 (Johnson et al. 1999).

Ground-based data from Walker (1992) for Reticulum and Brocato et al. (1996) for NGC 1786 and NGC 2210 already showed that these clusters were close in age to the Milky Way clusters. Here we revisit the question of the duration of the formation of metal-poor clusters in the LMC with HST WFPC2 data.

\section{Observations and Data Reduction}

For each cluster we have observations of $2 \times 260$ seconds and $3 \times 1000$ seconds in F555W and $2 \times 260$ and $4 \times 1000$ seconds in F814W. The long exposures were dithered. The data presented here are from the one chip centered on the cluster (PC for NGC 1786 and NGC 2210, WF3 for Reticulum). The frames were masked to eliminate the areas vignetted by the reimaging optics and a 
correction applied to renormalize all the pixels to the same effective area. Each frame was analysed with standard DAOPHOT/ALLSTAR procedures (Stetson 1987) to produce star lists for each frame. The frame-to-frame offsets were determined and the star lists combined to produce a master list of objects with the requirement that each object on the final list be found within 1 pixel (transformed coordinates) on at least 3 individual frames. This master candidate list was then the input to a run of ALLFRAME (Stetson 1994). The mean ALLFRAME magnitudes were then compared to magnitudes from $1 / 2$ " radius aperture photometry to derive an aperture correction. The calibration of Holtzman et al. (1997) was used to transform the HST data to $V$ and $I$ magnitudes. No CTE-correction has been made yet. For NGC 2210 and NGC 1786, stars closer than 3.3 " from the center have been eliminated because severe crowding was affecting the photometry.

\section{Results and Conclusions}

In Figure 1, we present the $V, V-I$ CMDs for NGC 1786, NGC 2210, and Reticulum. To compare these clusters with the LMC clusters previously studied, we have plotted the fiducial from NGC 2257, an old cluster with similar metallicity from Johnson et al. (1999). This fiducial has been shifted in $V$ and $V-I$ to account for different distances and reddening until the best fit by eye was obtained. That this fit matched the cluster sequences from the main sequence through the subgiant branch and the red giant branch indicates that the age of NGC 2257 is close to the ages of these clusters. Since this is a qualitative analysis, the errors at present are \pm 2 Gyr.

Reticulum, NGC 1786, and NGC 2210 were formed within 2 Gyr of the other old LMC clusters that have been studied so far. This indicates a relatively short burst of star cluster formation at the time the old Milky Way clusters were forming, across a large area of the LMC, an area far larger than today's optical bar.

\section{References}

Brocato, E., Castellani, V., Ferraro, F. R., Piersimoni, A. M., \& Testa, V. 1996, MNRAS, 282, 614

Holtzman, J., Burrows, C., Casertano, S., Hester, J., Trauger, J., Watson, A., \& Worthey, G. 1995, PASP, 1071065

Johnson, J. A., Bolte, M., Stetson, P. B., Heser, J. E., \& Sommerville, R. S. 1999, ApJ, 527, 199

Olsen, K. A. G., Hodge, P. W., Mateo, M., Olszewski, E. W., Schommer, R. A., Suntzeff, N. B., \& Walker, A. R. 1998, MNRAS, 300, 665

Olszewski, E. W., Schommer, R. A., Suntzeff, N. B., \& Harris, H. C. 1991, AJ, 101,515

Stetson, P. 1987, PASP, 99, 191

Stetson, P. 1994, PASP, 106, 250

Walker, A. 1992, AJ, 103, 1166 